H. UEDA

KODAI MATH. J.

4 (1981), 298-314

\title{
ON THE MINIMUM MODULUS OF A SUBHARMONIC OR AN ALGEBROID FUNCTION OF $\mu_{*}<1 / 2$
}

\author{
By Hideharu UEdA
}

0. Introduction. Let $y(z)$ be an $N$-valued entire algebroid function defined by an irreducible equation

$$
F(z, y)=y^{N}+A_{1}(z) y^{N-1}+\cdots+A_{N}(z)=0 .
$$

Denoting the $j$-th determination of $y$ by $y_{\jmath}$, we set

$$
M(r, y)=\max _{|z|=r} \max _{1 \leqq j \leqq N}\left|y_{j}(z)\right|, \quad m *(r, y)=\min _{|z|=r} \max _{1 \leqq j \leqq N}\left|y_{j}(z)\right| .
$$

Let $A$ be the system $\left(1, A_{1}, \cdots, A_{N}\right)$ and put

$$
B(z)=\max _{1 \leqq j \leqq N}\left|A_{j}(z)\right|, \quad M(r, B)=\max _{|z|=r} B(z), \quad m^{*}(r, B)=\min _{|z|=r} B(z) .
$$

Then Ozawa [12] showed that

$$
\frac{N \log ^{+} m^{*}(r, y)}{\log M(r, y)} \geqq \frac{\log m^{*}(r, B)+O(1)}{\log M(r, B)+O(1)} .
$$

And he obtained the following theorem by making use of Kjellberg's method [10].

THEOREM A. Let $y(z)$ be an $N$-valued entire algebroid function of lower order $\mu, 0 \leqq \mu<1 / 2$. Then

$$
\varlimsup_{r \rightarrow \infty} \frac{N^{2} \log m^{*}(r, y)}{\log M(r, y)} \geqq \cos \pi \mu .
$$

We can improve his result by two different methods. The first method is due to Baernstein [3]. He proved there

THEOREM B. Let $f$ be a nonconstant entre function. Let $\beta$ and $\lambda$ be numbers with $0<\lambda<\infty, 0<\beta \leqq \pi, \beta \lambda<\pi$. Then either

(a) there exist arbitrarily large values of $r$ for which the set of $\theta$ satisfying $\log \left|f\left(r e^{i \theta}\right)\right|>\cos \beta \lambda \log M(r, f)$ contains an interval of length at least $2 \beta$, or else

(b) $\lim _{r \rightarrow \infty} r^{-\lambda} \log M(r, f)$ exists, and is positive or $\infty$.

Received March 10, 1980 
It turns out by a minute observation of his papers [2], [3] that Theorem B still holds when we replace $|f|, \log \left|f\left(r e^{\imath \theta}\right)\right|$ and $\log M(r, f)$ by $B(z), \log B\left(r e^{\imath \theta}\right)$ and $\log M(r, B)$, respectively. Hence choosing $\beta=\pi$ and $\lambda=\mu+\varepsilon$ in Theorem $\mathrm{B}$, it follows from (2), Theorem $B$ and the above remark that

$$
\varlimsup_{r \rightarrow \infty} \frac{N \log m^{*}(r, y)}{\log M(r, y)} \geqq \cos \pi \mu .
$$

The second method is to make use of the notion of a local form of the Phragmén-Lindelöf indicator. This notion was introduced by Edrei [7] and is closely related to Pólya peaks. Drasin and Shea [6] proved that Pólya peaks of order $\rho$ exist if and only if $\rho \in\left[\mu_{*}, \lambda_{*}\right], \rho<\infty$, where

$$
\mu_{*}=\mu_{*}(T)=\inf \left\{\rho: \lim _{r_{,} \rightarrow \infty} \frac{T(C r, A)}{C^{\rho} T(r, A)}=0\right\},
$$

$$
\lambda_{*}=\lambda_{*}(T)=\sup \left\{\rho: \lim _{r, \overline{C \rightarrow \infty}} \frac{T(C r, A)}{C^{\rho} T(r, A)}=\infty\right\} .
$$

It is easy to see that $\mu_{*} \leqq \mu \leqq \lambda \leqq \lambda_{*}$, where $\lambda$ and $\mu$ are the order and the lower order of $T$, respectively. Edrei defined a local indicator for a sequence $\left\{f_{m}(z)\right\}_{1}^{\infty}$ of analytic functions such that $f_{m}(z)$ is regular and single-valued in the annulus: $r_{m}^{\prime} \leqq|z| \leqq r_{m}^{\prime \prime}(m=1,2, \cdots)$. However, his definition is naturally extended for a sequence $\left\{B_{m}(z)\right\}_{1}^{\infty}$ of subharmonic functions. Exact definition of the local indicator for a sequence $\left\{B_{m}(z)\right\}_{1}^{\infty}$ will be stated in $\S 1$. In $\S 2$, we shall state some elementary facts on subharmonic functions defined in $\boldsymbol{C}$. In $\S 3$ we shall prove the following Theorem 1. The case when $u(z)=\log |f(z)|$, and $f(z)$ is entire, is due to Edrei [7, Theorem 1]. In what follows, for a subharmonic function $u$ in $\boldsymbol{C}$, we put

$$
N(r, u)=\frac{1}{2 \pi} \int_{-\pi}^{+\pi} u\left(r e^{\imath \theta}\right) d \theta, \quad M(r, u)=\sup _{-\pi \leq \theta \leq \pi} u\left(r e^{\imath \theta}\right), \quad m^{*}(r, u)=\inf _{-\pi \leq \theta \leq \pi} u\left(r e^{\imath \theta}\right) .
$$

THEOREM 1. Let $u(z)$ be a nonconstant subharmonic function in $C$ and let $T(r, u)=N\left(r, u^{+}\right)$. Assume that $\mu_{*}=\mu_{*}(T)<1$. Let $\left\{r_{m}\right\}_{1}^{\infty}$ be a sequence of Pólya peaks of order $\rho\left(\mu_{*} \leqq \rho \leqq \lambda_{*}, 0<\rho<1\right)$ of $T(r, u)$. Then given $\varepsilon>0$, it is possible to find $a$ bound $s=s(\boldsymbol{\varepsilon})>0$, independent of $m$, and such that, in $\bigcup_{m=1}^{\infty}\left[r_{m} e^{-s}, r_{m} e^{s}\right]$ there exist arbitrarily large values of $r$ satısfying the inequality:

$$
m^{*}(r, u)>(\cos \pi \rho-\varepsilon) M(r, u) .
$$

COROLlary 1. Let $y(z)$ be an N-valued entire algebroid function and have $\mu_{*}<1 / 2$. Let $\left\{r_{m}\right\}_{1}^{\infty}$ be a sequence of Pólya peaks of order $\rho$ of $T(r, y)\left(\mu_{*} \leqq \rho \leqq \lambda_{*}\right)$ and let $0<\rho<1 / 2$. Then given $\varepsilon>0$, it is possible to find a bound $s=s(\varepsilon)$, independent of $m$, and such that, in $\bigcup_{m=1}^{\infty}\left[r_{m} e^{-s}, r_{m} e^{s}\right]$ there exist arbitrarily large values 
of $r$ satisfying the inequality:

$$
N \log m^{*}(r, y)>(\cos \pi \rho-\varepsilon) \log M(r, y) .
$$

This is also an improvement of Theorem A. However, since $\mu_{*} \leqq \mu$ (Equality does not alway's hold.), the second method is superior to the first one for this problem.

It is natural to consider an analogous problem to Theorem 1 for $\delta$-subharmonic functions-differences of subharmonic functions. That is, for a $\delta$-subharmonic function $v(z)=u^{(1)}(z)-u^{(2)}(z)$ of $\mu_{*}<1 / 2$, what can we say about the relation between $m^{*}(r, v)=\inf _{-\pi \leqq \theta \leqq \pi} v\left(r e^{i \theta}\right)$ and $T(r, v)=N\left(r, v^{+}\right)+N\left(r, u^{(2)}\right)$ ? In [1], Anderson and Baernstein considered a more general problem for $\delta$-subharmonic functions. The following theorem is a part of their consideration. Here we put for a $\delta$ subharmonic function $v=u^{(1)}-u^{(2)}$ in $C$

$$
\delta(\infty, v)=1-\varlimsup_{r \rightarrow \infty} \frac{N\left(r, u^{(2)}\right)}{T(r, v)} .
$$

THEOREM B. Let $v(z)=u^{(1)}(z)-u^{(2)}(z)$ be a $\delta$-subharmonic function in $\boldsymbol{C}$ of lower order $\mu, 0 \leqq \mu<1 / 2$. And assume that $\cos \pi \mu-1+\delta(\infty, v)>0$. Then it is possible to find a positive number $R$ and Pólya peak sequence $\left\{r_{m}\right\}_{1}^{\infty}$ of order $\mu$ of $T(r, v)$ satisfying the inequality:

$$
m^{*}\left(R r_{m}, v\right)>\left\{\frac{\pi \mu[\cos \pi \mu-(1-\delta(\infty, v))]}{\sin \pi \mu}-\varepsilon\right\} T\left(R r_{m}, v\right) .
$$

Using the concept of a local indicator, we can prove the following Theorem 2. The case when $v(z)=\log |f(z)|=\log \left|f_{1}(z)\right|-\log \left|f_{2}(z)\right|$, where $f=f_{1} / f_{2}$ is meromorphic, is due to Edrei [7, Theorem 2]. (The proof of Theorem 2 will be omitted.)

THEOREM 2. Let $v(z)=u^{(1)}(z)-u^{(2)}(z)$ be a $\delta$-subharmonic function in $\boldsymbol{C}$ and have $\mu_{*}<1 / 2$. Assume that $v(z)$ satisfies the following conditions (i) and (ii):

(i) $N\left(r, u^{(1)}\right) \sim T(r, v)(r \rightarrow \infty)$,

(ii) $\delta(\infty, v)+\cos \pi \rho-1=k>0$, where $\mu_{*} \leqq \rho \leqq \lambda_{*}, 0<\rho<1 / 2$.

And let $\left\{r_{m}\right\}_{1}^{\infty}$ be a sequence of Pólya peaks of order $\rho$ of $T(r, v)$. Then given $\varepsilon>0$, it is possible to find a bound $s=s(\varepsilon)>0$, independent of $m$, and such that, in $\bigcup_{m=1}^{\infty}\left[r_{m} e^{-s}, r_{m} e^{s}\right]$ there exist arbitrarily large values of $r$ satısfying the inequality:

$$
m^{*}(r, v)>\frac{\pi \rho(k-\varepsilon)}{\sin \pi \rho} T(r, v)
$$

COROLLARY 2. Let $y(z)$ be an $N$-valued algebrond function and have $\mu_{*}<1 / 2$. 
Assume that $\rho$ satisfies the following three conditions;
(i) $\mu_{*} \leqq \rho \leqq \lambda_{*}$,
(ii) $0<\rho<1 / 2$,
(iii) $\delta(\infty, y)+\cos \pi \rho-1=k>0$.

Let $\left\{r_{m}\right\}_{1}^{\infty}$ be a sequence of Pólya peaks of order $\rho$ of $T(r, y)$. Then given $\varepsilon>0$, it is possible to find a bound $s=s(\varepsilon)>0$, independent of $m$, and such that in $\bigcup_{m=1}^{\infty}\left[r_{m} e^{-s}, r_{m} e^{s}\right]$ there exist arbitrarily large values of $r$ satısfying the inequality:

$$
\log m^{*}(r, y)>\frac{\pi \rho(k-\varepsilon)}{\sin \pi \rho} T(r, y) .
$$

The derivation of Corollary 2 will be done in $\S 4$.

Finally, in $\S 5$, as another application of a local indicator, we shall show the following theorem.

THEOREM 3. Let $v=u^{(1)}-u^{(2)}$ be a $\delta$-subharmonic function in $\boldsymbol{C}$ and have $\mu_{*}<1 / 2$. Assume that $N\left(r, u^{(1)}\right) \sim T(r, v)(r \rightarrow \infty)$ and let $\rho$ satisfy the following three conditions:

(i) $\mu_{*} \leqq \rho \leqq \lambda_{*}$, (ii) $0<\rho<1 / 2$, (iii) $\cos \pi \rho-1+\delta(\infty, v) /(2-\delta(\infty, v))=k_{2}>0$.

Further let $\left\{r_{m}\right\}_{1}^{\infty}$ be a sequence of Pólya peaks of order $\rho$ of $T(r, v)$, and let

$$
m_{2}(r, v)=\left\{N\left(r, v^{2}\right)\right\}^{1 / 2} \text {. }
$$

Then given $\varepsilon>0$, it is possible to find a bound $s=s(\varepsilon)>0$, independent of $m$, and such that in $\bigcup_{m=1}^{\infty}\left[r_{m} e^{-s}, r_{m} e^{s}\right]$ there exist arbitrarily large values of $r$ satisfying the inequality:

$$
m^{*}(r, v)>\left\{\frac{k_{2}}{\sqrt{1 / 2+(\sin 2 \pi \rho) / 4 \pi \rho}}-\varepsilon\right\} m_{2}(r, v) .
$$

In particular, if $v$ is subharmonic, then the assumption: $N\left(r, u^{(1)}\right) \sim T(r, v)$ can be dropped.

If $\delta(\infty, v)=1$, the estimate (9) is best possible. For example, consider a subharmonic function :

$$
v(z)=\frac{\pi \gamma^{\rho}}{\sin \pi \rho} \cos \rho \theta .
$$

For an $N$-valued algebroid function $y(z)$, we introduce the following quantity:

$$
C(r, y)=\left[\frac{1}{2 \pi} \int_{-\pi}^{+\pi} \sum_{j=1}^{N}\left\{\log ^{+}\left|y_{j}\left(r e^{i \theta}\right)\right|\right\}^{2} d \theta\right]^{1 / 2}\left(y_{\jmath}: \jmath \text {-th determination of } y\right) .
$$


COROLlary 3. Let $y(z)$ be an $N$-valued algebroid function and have $\mu_{*}<1 / 2$. And let $\rho$ satisfy the following three conditions:

(i) $\mu_{*} \leqq \rho \leqq \lambda_{*}$, (ii) $0<\rho<1 / 2$, (iii) $k_{2}=\cos \pi \rho-1+\delta(\infty, y) /(2-\delta(\infty, y))>0$.

Further let $\left\{r_{m}\right\}_{1}^{\infty}$ be a sequence of Pólya peaks of order $\rho$ of $T(r, y)$. Then given $\varepsilon>0$, it is possible to find a bound $s=s(\varepsilon)>0$, independent of $m$, and such that in $\bigcup_{m=1}^{\infty}\left[r_{m} e^{-s}, r_{m} e^{s}\right]$ there exist arbitrarily large values of $r$ satısfying the inequality:

$$
N \log m^{*}(r, y)>\frac{k_{2}-\varepsilon}{\sqrt{1 / 2+(\sin 2 \pi \rho) / 4 \pi \rho}} C(r, y) .
$$

1. Definition of the local indicator of order $\rho$ of a sequence $\left\{B_{m}(z)\right\}_{1}^{\infty}$ of subharmonic functions.

(i) three infinite sequences of positive numbers $\left\{r_{m}^{\prime}\right\}_{1}^{\infty},\left\{r_{m}\right\}_{1}^{\infty},\left\{r_{m}^{\prime \prime}\right\}_{1}^{\infty}$ such that $r_{m}^{\prime}<r_{m}<r_{m}^{\prime \prime}<r_{m+1}^{\prime}(m=1,2, \cdots)$, and such that, as $m \rightarrow \infty$

$$
r_{m} / r_{m}^{\prime} \longrightarrow \infty, \quad r_{m}^{\prime \prime} / r_{m} \longrightarrow \infty .
$$

(ii) a sequence $\left\{B_{m}(z)\right\}_{1}^{\infty}$ such that $B_{m}(z)$ is subharmonic in the annulus: $r_{m}^{\prime}<|z|<r_{m}^{\prime \prime}$.

(iii) a strictly positive sequence $\left\{V\left(r_{m}\right)\right\}_{1}^{\infty}$ and a quantity $\rho(0<\rho<\infty)$. We then define a sequence $\left\{V_{m}(z)\right\}_{1}^{\infty}$ of analytic "comparison functions":

$$
V_{m}(z)=V_{m}(r) e^{\imath \rho \theta} \equiv V\left(r_{m}\right)\left(\frac{r}{r_{m}}\right) e^{\imath \rho \theta} \quad\left(z=r e^{\imath \theta}\right) .
$$

The symbol $V_{m}(r)$ always refers to the choice of $\theta=0$.

(iv) Consider the intervals $I_{m}=\left[r_{m}^{\prime}, r_{m}^{\prime \prime}\right](m=1,2, \cdots)$ as well as the intervals $I_{m}(s)=\left[r_{m} e^{-s}, r_{m} e^{s}\right](m=1,2, \cdots, s=1,2, \cdots)$, and let

$$
\Lambda=\bigcup_{m=1}^{\infty} I_{m}, \quad \Lambda(s)=\bigcup_{m=1}^{\infty} I_{m}(s) \quad(s=1,2, \cdots) .
$$

(v) Let the sequence $\left\{B_{m}(z)\right\}_{1}^{\infty}$ be chosen so that

$$
\varlimsup_{\substack{r \rightarrow \infty \\ r \in \Lambda}} \frac{M(r, B)}{V(r)}<\infty,
$$

where $B(z)$ stands for $B_{m}(z)$ in the annulus : $r_{m}^{\prime}<|z|<r_{m}^{\prime \prime} .(m=1,2, \cdots)$. We set for every real value of $\theta$,

and consider

$$
h_{s}(\theta)=\varlimsup_{\substack{r \rightarrow \infty \\ r \in \Lambda(s)}} \frac{B\left(r e^{2 \theta}\right)}{V(r)} \quad(s=1,2, \cdots),
$$

$$
h(\theta)=\lim _{s \rightarrow \infty} h_{s}(\theta) .
$$


The real function $h(\theta)$ is, by definition, the local indicator of order $\rho$ of $\left\{B_{m}(z)\right\}_{1}^{\infty}$ at the peaks $\left\{r_{m}\right\}_{1}^{\infty}$. With this definition, Edrei's Fundamental Lemma can be extended straightforwardly for the sequence $\left\{B_{m}(z)\right\}_{1}^{\infty}$ of subharmonic functions (For the proof, cf. [7, pp. 159-162]).

Fundamental Lemma. Let $h(\theta)$ be the local indicator of order $\rho(0<\rho<\infty)$ of $\left\{B_{m}(z)\right\}_{1}^{\infty}$ at the peaks $\left\{r_{m}\right\}_{1}^{\infty}$. Let $\theta_{1}, \theta_{2}$ be given such that $0<\theta_{2}-\theta_{1}<\pi / \rho$, and let the constants $a, b$ be such that the sinusord $H(\theta)=a \cos \rho \theta+b \sin \rho \theta$ satisfies the conditions $h\left(\theta_{1}\right) \leqq H\left(\theta_{1}\right), h\left(\theta_{2}\right) \leqq H\left(\theta_{2}\right)$. Then given $\varepsilon>0$ and any integer $s>0$, there exists a bound $r_{0}=r_{0}\left(\varepsilon, s, a, b, \theta_{1}, \theta_{2}\right)$, independent of $\theta$, such that for $r \in \Lambda(s), \theta_{1} \leqq \theta \leqq \theta_{2}, r \geqq r_{0}$

$$
B\left(r e^{\imath \theta}\right) \leqq(H(\theta)+\varepsilon) V(r) .
$$

From Fundamental Lemma, we immediately have $h(\theta) \leqq H(\theta)\left(\theta_{1} \leqq \theta \leqq \theta_{2}\right)$, that is, the subtrigonometric character of $h(\theta)$. It is known that many important properties of an indicator depend only on its subtrigonometric character (cf. [5]). For example, we have the following fact (cf. [5, pp. 42-45]).

Let $h(\theta)$ be the local indicator of order $\rho$ of $\left\{B_{m}(z)\right\}_{1}^{\infty}$. Assume that $h(\theta)$ $\not \equiv-\infty$, and let $\theta_{1}, \theta_{2}, \theta_{3}$ be such that $0<\theta_{2}-\theta_{1}<\pi / \rho, 0<\theta_{3}-\theta_{2}<\pi / \rho$. Then

$$
\left|\begin{array}{lll}
h\left(\theta_{1}\right) & \cos \rho \theta_{1} & \sin \rho \theta_{1} \\
h\left(\theta_{2}\right) & \cos \rho \theta_{2} & \sin \rho \theta_{2} \\
h\left(\theta_{3}\right) & \cos \rho \theta_{3} & \sin \rho \theta_{3}
\end{array}\right| \geqq 0 .
$$

In partıcular, if $0 \leqq \theta<\pi / \rho$, then

$$
\frac{h(-\theta)+h(\theta)}{2} \geqq h(0) \cos \rho \theta .
$$

2. Some elementary facts on subharmonic functons defined in $C$. Since we are interested in results for large values of $r$ in Theorem 1, we may assume that $u(z)$ is harmonic in a neighborhood of the orign. Further we may prove Theorem 1 for $u(0)=0$. In fact, assume that Theorem 1 is valid for an arbitrary subharmonic function $v(z)$ of $\mu_{*}<1 / 2$ which is harmonic in a neighborhood of $z=0$ and satisfies $v(0)=0$. Take an arbitrary subharmonic function $u(z)$ of $\mu_{*}<1 / 2$ which is harmonic in a neighborhood of $z=0$. Put $v(z)=u(z)-u(0)$. By the Riesz representation theorem there exists a positive Borel measure $\nu$ and $C$ such that for $|z|<R(0<R<\infty)$

$$
\begin{aligned}
v(z) & =h(z)+\int_{|\zeta|<R} \log |z-\zeta| d \nu(\zeta) \\
& =h(z)+\int_{|\zeta|<R} \log |\zeta| d \nu(\zeta)+\int_{|\zeta|<R} \log \left|1-\frac{z}{\zeta}\right| d \nu(\zeta),
\end{aligned}
$$


where $h(z)$ is harmonic in $|z|<R$. Let $n(r)=\nu(|\zeta|<r)$. Then Jensen's formula for subharmonic functions (cf. [9]) gives

$$
N(r, v)=\frac{1}{2 \pi} \int_{-\pi}^{+\pi} v\left(r e^{\imath \theta}\right) d \theta=\int_{0}^{r} \frac{n(t)}{t} d t \leqq T(r, v) \leqq M(r, v),
$$

which implies that " $N(r, v)$ is bounded. $\Leftrightarrow v(z)$ is harmonic in $C$." Assume now that $T(r, v)$ is bounded. Then $v^{+}$is harmonic in $\boldsymbol{C}$. Since $v^{+} \geqq 0$, this shows that $v^{+}$is a constant. Therefore $N(r, v)$ is bounded, so that $v$ is harmonic in $\boldsymbol{C}$. However, since $v$ is bounded above, $v$ must be a constant. Hence the nonconstancy of $v$ implies that $T(r, v) / \infty$ and that $M(r, v) / \infty(r \rightarrow \infty)$. Thus there exists a $r_{0}=r_{0}(\varepsilon)>0$ such that $r \geqq r_{0}$ implies

$$
|u(0)|\left(1-\cos \pi \rho+\frac{\varepsilon}{2}\right)<\frac{\varepsilon}{2} M(r, v) .
$$

Further by the above assumption, there exists a sequence $\left\{\chi_{n}\right\}_{1}^{\infty} / \infty$ contained in $\bigcup_{m=1}^{\infty}\left[r_{m} e^{-s}, r_{m} e^{s}\right]\left(\left\{r_{m}\right\}_{1}^{\infty}\right.$ : a Pólya peak sequence of order $\rho$ of $T(r, u) ; s=s(\varepsilon)$ : a positive integer) satisfying the inequality:

$$
m^{*}\left(\chi_{n}, v\right)>\left(\cos \pi \rho-\frac{\varepsilon}{2}\right) M\left(\chi_{n}, v\right) .
$$

It follows from (13) and (14) that

$$
m^{*}\left(\chi_{n}, u\right)>(\cos \pi \rho-\varepsilon) M\left(\chi_{n}, u\right) \quad\left(\chi_{n} \geqq r_{0}\right) .
$$

In what follows we may assume that $u(z)$ is harmonic in a neighborhood of 0 and satisfies $u(0)=0$.

Now, we put

$$
\begin{aligned}
& u_{1}(z, R)=\int_{|\zeta|<R} \log \left|1-\frac{z}{\zeta}\right| d \nu(\zeta), \\
& u_{2}(z, R)=\int_{|\zeta|<R} \log \left|1+\frac{z}{|\zeta|}\right| d \nu(\zeta)=\int_{0}^{R} \log \left|1+\frac{z}{t}\right| d n(t) .
\end{aligned}
$$

Then $u_{1}(z, R)$ and $u_{2}(z, R)$ are subharmonic in $C$ and they satisfy

$$
m^{*}\left(r, u_{2}\right) \leqq m^{*}\left(r, u_{1}\right) \leqq M\left(r, u_{1}\right) \leqq M\left(r, u_{2}\right) .
$$

Next, let

$$
u_{3}(z, R)=u(z)-u_{1}(z, R) .
$$

Then, using (17), (11), and (15), we have

$$
u_{3}(z, R)=h(z)+\int_{|\zeta|<R} \log |\zeta| d \nu(\zeta) \quad(|z|<R) .
$$


which shows that $u_{3}(z, R)$ is harmonic in $|z|<R$. Let $f(z)$ be regular in $|z|<R$ such that $\operatorname{Re} f(z)=u_{3}(z, R)$ and $f(0)=0$. Hence by a theorem of Carathéodory

$$
|f(z)| \leqq \frac{2|z|}{R-|z|} M\left(R, u_{3}\right) \quad(|z|<R) .
$$

Further, an estimate due to Kjellberg $[10$, p. 92$]$ or Barry $[4$, p. 182] gives

$$
M\left(2 R, u_{3}\right) \leqq M(2 R, u) .
$$

Combining (18) and (19) we obtain

$$
\left|u_{3}(z, R)\right| \leqq|f(z)|<\frac{4 M(2 R, u)}{R} r \quad\left(|z|=r<\frac{R}{2}\right) .
$$

3. Proof of Theorem 1. Since we are mainly interested in Corollary 1, we shall prove only for the case of $\mu_{*}<1 / 2, \mu_{*} \leqq \rho \leqq \lambda_{*}$ and $0<\rho<1 / 2$. Let $\left\{r_{m}\right\}_{1}^{\infty}$ be a sequence of Pólya peaks of order $\rho$ of $T(r, u)$. And let $\left\{r_{m}^{\prime}\right\}_{1}^{\infty},\left\{r_{m}^{\prime \prime}\right\}_{1}^{\infty},\left\{\varepsilon_{m}\right\}_{1}^{\infty}$ be the associated sequences with Pólya peaks $\left\{r_{m}\right\}_{1}^{\infty}$ of order $\rho$. Choose $\left\{V\left(r_{m}\right)\right\}_{1}^{\infty}$ as follows.

$$
V\left(r_{m}\right)=\left(1+\varepsilon_{m}\right) T\left(r_{m}, u\right) \quad(m=1,2, \cdots) .
$$

This implies

$$
T(r, u)<V(r) \quad(r \in \Lambda) .
$$

Put

$$
B_{m}(z)=u_{2}\left(z, r_{m}^{\prime \prime} / 4\right)=\int_{0}^{r_{m^{*}} / 4} \log \left|1+\frac{z}{t}\right| d n(t) \quad\left(r_{m}^{\prime} \leqq|z| \leqq r_{m}^{\prime \prime}\right)
$$

and we consider the local indicator $h(\theta)$ of order $\rho$ of $\left\{B_{m}(z)\right\}_{1}^{\infty}$ at the peaks $\left\{r_{m}\right\}_{1}^{\infty}$.

(i) Existence of $h(\theta)$ : By definition we may show that

$$
\varlimsup_{\substack{r \rightarrow \infty \\ r \in \Lambda}} \frac{B(r)}{V(r)}<\infty .
$$

Put

$$
n_{m}^{(0)}(t)=\left\{\begin{array}{l}
n(t) \quad\left(t \leqq \frac{r_{m}^{\prime \prime}}{4}\right) \\
n\left(\frac{r_{m}^{\prime \prime}}{4}\right)\left(t>\frac{r_{m}^{\prime \prime}}{4}\right)
\end{array}, \quad N_{m}^{(0)}(t)=\int_{0}^{t} \frac{n_{m}^{(0)}(r)}{r} d r\right.
$$

Then 


$$
\begin{aligned}
B_{m}(r) & =\int_{0}^{\infty} \log \left(1+\frac{r}{t}\right) d n_{m}^{(0)}(t)=r \int_{0}^{\infty} \frac{n_{m}^{(0)}(t)}{(t+r) t} d t=r \int_{0}^{\infty} \frac{d N_{m}^{(0)}(t)}{t+r} \\
& =r \int_{0}^{\infty} \frac{N_{m}^{(0)}(t)}{(t+r)^{2}} d t=r\left(\int_{0}^{r_{m}^{\prime}}+\int_{r_{m}^{\prime}}^{r_{m}^{\prime \prime} / 4}+\int_{r_{m^{\prime}} / 4}^{\infty}\right) \frac{N_{m}^{(0)}(t)}{(t+r)^{2}} d t
\end{aligned}
$$

Since

$$
N_{m}^{(0)}(t)=\left\{\begin{array}{l}
N(t) \quad\left(t \leqq \frac{r_{m}^{\prime \prime}}{4}\right) \\
N\left(\frac{r_{m}^{\prime \prime}}{4}\right)+n\left(\frac{r_{m}^{\prime \prime}}{4}\right) \log \left(\frac{4 t}{r_{m}^{\prime \prime}}\right) \quad\left(t>\frac{r_{m}^{\prime \prime}}{4}\right),
\end{array}\right.
$$

we easily obtain

$$
B_{m}(r) \leqq \frac{r_{m}^{\prime}}{r} N\left(r_{m}^{\prime}\right)+A \frac{r}{r_{m}^{\prime \prime}} N\left(r_{m}^{\prime \prime}\right)+r \int_{r_{m}^{\prime \prime}}^{r_{m^{\prime \prime}} / 4} \frac{N(t)}{(t+r)^{2}} d t
$$

By (22) we have

$$
\text { ( } A \text { : an absolute constant). }
$$

Thus for $r \in I_{m}$

$$
N(r, u) \leqq T(r, u)<V(r) \quad\left(r \in I_{m}\right)
$$

$$
\begin{aligned}
B_{m}(r) & \leqq \frac{r_{m}^{\prime}}{r} V\left(r_{m}^{\prime}\right)+A \frac{r}{r_{m}^{\prime \prime}} V\left(r_{m}^{\prime \prime}\right)+r \int_{r_{m}^{\prime}}^{r_{m}^{\prime \prime}} \frac{V(t)}{(t+r)^{2}} d t \\
& \leqq\left(\frac{r_{m}^{\prime}}{r}\right) V(r)\left(\frac{r_{m}^{\prime}}{r}\right)^{\rho}+A\left(\frac{r}{r_{m}^{\prime \prime}}\right) V(r)\left(\frac{r_{m}^{\prime \prime}}{r}\right)^{\rho}+V(r) \int_{0}^{\infty} \frac{x^{\rho}}{(1+x)^{2}} d x \\
& <V(r)\left[1+A+\int_{0}^{\infty} \frac{x^{\rho}}{(1+x)^{2}} d x\right] .
\end{aligned}
$$

This shows (24).

(ii) $h(0) \geqq 1$ : By definition we may prove

$$
\varliminf_{m \rightarrow \infty} \frac{B\left(r_{m}\right)}{V\left(r_{m}\right)} \geqq 1 \text {. }
$$

From (21), (17), (16), (23) and (20) it follows that

$$
\begin{aligned}
\frac{V\left(r_{m}\right)}{1+\varepsilon_{m}} & =T\left(r_{m}, u\right) \leqq M\left(r_{m}, u\right) \leqq M\left(r_{m}, u_{1}\right)+M\left(r_{m}, u_{3}\right) \\
& \leqq M\left(r_{m}, u_{2}\right)+M\left(r_{m}, u_{3}\right) \leqq B\left(r_{m}\right)+\frac{4 M\left(r_{m}^{\prime \prime} / 2, u\right)}{r_{m}^{\prime \prime} / 4} r_{m} \\
& =B\left(r_{m}\right)+16 M\left(r_{m}^{\prime \prime} / 2, u\right) \frac{r_{m}}{r_{m}^{\prime \prime}}
\end{aligned}
$$




$$
\leqq B\left(r_{m}\right)+48 T\left(r_{m}^{\prime \prime}, u\right) \frac{r_{m}}{r_{m}^{\prime \prime}} \leqq B\left(r_{m}\right)+48 V\left(r_{m}\right)\left(\frac{r_{m}}{r_{m}^{\prime \prime}}\right)^{1-\rho}
$$

where we used the fact that $M(r, u) \leqq 3 T(2 r, u)$ (cf. [9, Chapter 3]). Since $r_{m} / r_{m}^{\prime \prime}$ $\rightarrow 0$ as $m \rightarrow \infty$, (25) follows.

(iii) By (23) we have $B_{m}\left(r e^{\imath \theta}\right)=B_{m}\left(r e^{-\imath \theta}\right)$ for $0 \leqq \theta \leqq \pi$, which implies $h(\theta)=$ $h(-\theta)(0 \leqq \theta \leqq \pi)$. It follows from this, (10) and (ii) that

$$
h(\theta) \geqq h(0) \cos \rho \theta \geqq \cos \rho \theta>0 .
$$

(iv) By (17), (16), (20) and (22) we have for $r \leqq r_{m}^{\prime \prime} / 8$

$$
\begin{aligned}
m^{*}(r, u) & \geqq m^{*}(r, u)+m^{*}\left(r, u_{3}\right) \\
& \geqq m^{*}\left(r, u_{2}\right)-\frac{4 M\left(r_{m}^{\prime \prime} / 2, u\right)}{r_{m}^{\prime \prime} / 4} r \geqq m^{*}\left(r, u_{2}\right)-48 V\left(r_{m}^{\prime \prime}\right) \frac{r}{r_{m}^{\prime \prime}} \\
& =m^{*}\left(r, u_{2}\right)-48\left(\frac{r}{r_{m}^{\prime \prime}}\right)^{1-\rho} V(r) .
\end{aligned}
$$

In the same way we obtain

$$
M(r, u) \leqq M\left(r, u_{2}\right)+48\left(\frac{r}{r_{m}^{\prime \prime}}\right)^{1-\rho} V(r) \quad\left(r \leqq r_{m}^{\prime \prime} / 8\right)
$$

(v) For given $\eta>0$ (small enough), choose $s$ (a positive integer) such that $h_{s}(\pi)>h(\pi)-\eta$. By the definition of $h_{s}(\pi)$, there exists a sequence $\left\{\chi_{n}\right\}_{1}^{\infty}\left(\Gamma_{\infty}\right)$ $\subset \bigcup_{m=1}^{\infty}\left[r_{m} e^{-s}, r_{m} e^{s}\right]$ satisfying $B\left(-\chi_{n}\right)>\left(h_{s}(\pi)-\eta\right) V\left(\chi_{n}\right)>(h(\pi)-2 \eta) V\left(\chi_{n}\right)$. Hence by (27) and (26)

$$
\begin{aligned}
m^{*}\left(\chi_{n}, u\right) & >(h(\pi)-3 \eta) V\left(\chi_{n}\right) \quad\left(n \geqq n_{0}(\eta, s)\right) \\
& >(h(0) \cos \pi \rho-3 \eta) V\left(\chi_{n}\right) \geqq(\cos \pi \rho-3 \eta) V\left(\chi_{n}\right)
\end{aligned}
$$

We may assume that $\cos \pi \rho-3 \eta>0$. On the other hand, by the definition of $h(0)$ and (28)

$$
M\left(\chi_{n}, u\right)<(h(0)+\eta) V\left(\chi_{n}\right)+\eta V\left(\chi_{n}\right) \quad\left(n \geqq n_{1}(\eta, s)\right) .
$$

It follows from (29) and (30) that

$$
\frac{m^{*}\left(\chi_{n}, u\right)}{M\left(\chi_{n}, u\right)}>\frac{h(\pi)-3 \eta}{h(0)+2 \eta}>\cos \pi \rho-\varepsilon
$$

Proof of Corollary 1. Let $y(z)$ be an $N$-valued entire algebroid function defined by (1). And let $A$ be the system $\left(1, A_{1}, \cdots, A_{N}\right)$. Then Valiron [13] proved that 


$$
T(r, A)=N T(r, y)+O(1) .
$$

Next, put $u(z)=\log B(z)=\max _{1 \leqq j \leqq N} \log \left|A_{j}(z)\right|$. Evidently

$$
T(r, A)=N\left(r, u^{+}\right)=T(r, u) .
$$

From (31) and (32) we deduce that $\left\{r_{m}\right\}_{1}^{\infty}$ is a Pólya peak sequence of order $\rho$ of $T(r, u)(\rho<1 / 2)$. Hence Theorem 1 implies the existence of a positive integer $s=s(\varepsilon)$ and a sequence $\left\{\chi_{n}\right\}_{1}^{\infty} / \infty$ contained in $\Lambda(s)$ such that

$$
\log m^{*}\left(\chi_{n}, B\right)>(\cos \pi \rho-\varepsilon) \log M\left(\chi_{n}, B\right) \quad(n=1,2, \cdots) .
$$

Combining this and (2), we have the desired result.

4. Proof of Carollary 2. Let $y(z)$ be an $N$-valued algebroid function defined by the irreducible equation

$$
F(z, y)=A_{0}(z) y^{N}+\cdots+A_{N}(z)=0 .
$$

And let $A=\left(A_{0}, \cdots, A_{N}\right)$. Then

$$
\min _{|z|=r} \max _{1 \leqq j \leqq N} \log \left|A_{j}(z) / A_{0}(z)\right| \leqq N \log ^{+} m^{*}(r, y)+O(1) .
$$

For the proof, cf. [12, p. 167]. Since (31) holds also in this case, we have

$$
\frac{\log ^{+} m^{*}(r, y)}{T(r, y)} \geqq \frac{\min _{|z|=r} \max _{1 \leq j \leq N} \log ^{+}\left|A_{j}(z) / A_{0}(z)\right|+O(1)}{T(r, A)+O(1)} .
$$

Now, let $v=u^{(1)}-u^{(2)}$, where $u^{(1)}(z)=\max _{0 \leqq j \leqq N} \log \left|A_{\jmath}(z)\right|, u^{(2)}(z)=\log \left|A_{0}(z)\right|$. Then it is clear that

$$
\begin{aligned}
T(r, v) & =N\left(r, v^{+}\right)+N\left(r, u^{(2)}\right)=N(r, v)+N\left(r, u^{(2)}\right) \\
& =N\left(r, u^{(1)}\right)=T(r, A)=N T(r, y)+O(1),
\end{aligned}
$$

and

$$
1-\delta(\infty, v)=\varlimsup_{r \rightarrow \infty} \frac{N\left(r, u^{(2)}\right)}{T(r, v)}=\varlimsup_{r \rightarrow \infty} \frac{N\left(r, 0, A_{0}\right)}{T(r, A)}=\varlimsup_{r \rightarrow \infty} \frac{N(r, \infty, y)}{T(r, y)}=1-\delta(\infty, y) .
$$

We deduce from (34) that $\left\{r_{m}\right\}_{1}^{\infty}$ is a sequence of Pólya peaks of order $\rho$ of $T(r, v)$. Further note that the condition (ii) in Theorem 2 follows from (35) and the condition (iii). Hence Theorem 2 guarantees the existence of a positive integer $s=s(\varepsilon)$ and a sequence $\left\{\chi_{n}\right\}_{1}^{\infty} \subset \Lambda(s)$ tending to $\infty$ such that

$$
\min _{|z|=r} \max _{1 \leq j \leq N} \log ^{+}\left|A_{\jmath}(z) / A_{0}(z)\right|>\frac{\pi \rho(k-\varepsilon)}{\sin \pi \rho} T\left(\chi_{n}, A\right) .
$$

Combinig this and (33) we have the desired result. 
5. Proof of Theorem 3. We may assume that $u^{(1)}$ and $u^{(2)}$ are harmonic in a neighborhood of 0 and that $u^{(1)}(0)=u^{(2)}(0)=0$. In fact, assume that Theorem 3 holds for the set $\mathscr{I}$ of such $\delta$-subharmonic functions. Take an arbitrary nonconstant $\delta$-subharmonic function $v=u^{(1)}-u^{(2)}$ satisfying the assumption of Theorem 3. Since we are interested in results for large values of $r$, we may assume that $u^{(1)}$ and $u^{(2)}$ are harmonic in a neighborhood of 0 . Next put $\tilde{u}^{(1)}(z)=u^{(1)}(z)$ $-u^{(1)}(0), \tilde{u}^{(2)}(z)=u^{(2)}(z)-u^{(2)}(0)$, and $\tilde{v}=\tilde{u}^{(1)}-\tilde{u}^{(2)}$. Since nonconstancy of $v$ implies that $T(r, v) \nearrow \infty$ as $r \rightarrow \infty$ (For the proof, cf. $\S 2$ ), we easily have

$$
T(r, v)=T(r, \tilde{v})+O(1)=(1+o(1)) T(r, \tilde{v}) \quad(r \rightarrow \infty) .
$$

From (36), if $\left\{r_{m}\right\}_{1}^{\infty}$ is a sequence of Pólya peaks of order $\rho$ of $T(r, v)$, it is also a Pólya peak sequence of order $\rho$ of $T(r, \tilde{v})$. Further evidently (36) implies that "All the assumptions of Theorem 3 are satisfied for $v(z)$. All the assumptions of Theorem 3 are satisfied for $\tilde{v}(z)$." Hence by assumption Theorem 3 guarantees the existence of a positive integer $s=s(\varepsilon)>0$ and a sequence $\left\{\chi_{n}\right\}_{1}^{\infty} \subset \Lambda(s)$ tending to $\infty$ such that

$$
\left.m^{*}\left(\chi_{n}, \tilde{v}\right)>\frac{k_{2}-\varepsilon / 2}{\sqrt{1 / 2+\sin 2 \pi \rho / 4 \pi \rho}} m_{2}\left(\chi_{n}, \tilde{v}\right) \quad n=1,2, \cdots\right) .
$$

Next, it is clear that

$$
m^{*}\left(\chi_{n}, v\right)=m^{*}\left(\chi_{n}, \tilde{v}\right)+u^{(1)}(0)-u^{(2)}(0),
$$

and

$$
m_{2}(r, v) \geqq N(r,|v|)=N\left(r, v^{+}\right)+N\left(r, v^{-}\right)=2 T(r, v)-N\left(r, u^{(1)}\right)-N\left(r, u^{(2)}\right) .
$$

It follows from (39) and $\delta(\infty, v)>0$ that $m_{2}(r, v) \rightarrow \infty$ as $r \rightarrow \infty$. Since $\tilde{v}=$ $v-\left(u^{(1)}(0)-u^{(2)}(0)\right) \equiv v-c$, we have

$$
m_{2}^{2}(r, \tilde{v})=m_{2}^{2}(r, v-c) \geqq m_{2}^{2}(r, v)+c^{2}-2|c| m_{2}(r, v)=\left\{m_{2}(r, v)-|c|\right\}^{2},
$$

so that

$$
m_{2}(r, \tilde{v}) \geqq m_{2}(r, v)-|c| \quad\left(r \geqq r_{0}(|c|)\right) .
$$

Now, noting that $m_{2}(r, v) \rightarrow \infty$ as $r \rightarrow \infty$, there exists a $r_{1}>0$ such that $r \geqq r_{1}$ implies

$$
|c|\left(1+\frac{k_{2}-\varepsilon / 2}{\sqrt{1 / 2+\sin 2 \pi \rho / 4 \pi \rho}}\right)<\frac{\varepsilon}{2 \sqrt{1 / 2+\sin 2 \pi \rho / 4 \pi \rho}} m_{2}(r, v) .
$$

Combining (37), (38), (40) and (41) we deduce

$$
m^{*}\left(\chi_{n}, v\right)>\frac{k_{2}-\varepsilon}{\sqrt{1 / 2+\sin 2 \pi \rho / 4 \pi \rho}} m_{2}\left(\chi_{n}, v\right) \quad\left(n \geqq n_{0}\right) .
$$

From now on, we assume that $v \in \mathscr{F}$. Let $\nu^{(j)}$ be the Riesz mass associated with 
$u^{(j)}(\jmath=1,2)$, and let $n^{(j)}(t)=\nu^{(j)}(|\zeta|<t)$. Further let $\left\{r_{m}^{\prime}\right\}_{1}^{\infty},\left\{r_{m}^{\prime \prime}\right\}_{1}^{\infty},\left\{\varepsilon_{m}\right\}_{1}^{\infty}$ be the associated sequences with $\left\{r_{m}\right\}_{1}^{\infty}$. Choose $V\left(r_{m}\right)=\left(1+\varepsilon_{m}\right) T\left(r_{m}, v\right)$, which implies

$$
T(r, v)<V(r) \quad(r \in \Lambda) .
$$

Put

$$
B_{m}(z)=u_{2}^{(1)}\left(z, r_{m}^{\prime \prime} / 4\right)+u_{2}^{(2)}\left(z, r_{m}^{\prime \prime} / 4\right)=\int_{0}^{r_{m}^{\prime} / 4} \log \left|1+\frac{z}{t}\right| d\left\{n^{(1)}(t)+n^{(2)}(t)\right\}
$$

Now, we consider the local indicator $h(\theta)$ of order $\rho$ of $\left\{B_{m}(z)\right\}_{1}^{\infty}$ at the peaks $\left\{r_{m}\right\}_{1}^{\infty}$. As in the proof of Theorem 1 , we can easily see the existence of $h(\theta)$. Here we shall show $h(0) \geqq 1$. By our assumptions, as $m \rightarrow \infty$

$$
\frac{V\left(r_{m}\right)}{1+\varepsilon_{m}}=T\left(r_{m}, v\right) \sim N\left(r_{m}, u^{(1)}\right)=N\left(r_{m}, u_{2}^{(1)}\right) \leqq N\left(r_{m}, u_{2}^{(1)}+u_{2}^{(2)}\right) \leqq B_{m}\left(r_{m}\right) .
$$

Hence by the definition of $h(0)$

$$
h(0) \geqq \varliminf_{m \rightarrow \infty} \frac{B_{m}\left(r_{m}\right)}{V\left(r_{m}\right)} \geqq 1 .
$$

Next, using (17) we have

$$
\begin{aligned}
v(z) & =u^{(1)}(z)-u^{(2)}(z)=u_{1, m}^{(1)}(z)-u_{1, m}^{(2)}(z)+u_{3, m}^{(1)}(z)-u_{3, m}^{(2)}(z) \\
& \equiv u_{1, m}^{(1)}(z)-u_{1, m}^{(2)}(z)+W_{m}(z) .
\end{aligned}
$$

Since $W_{m}(z)$ is harmonic in $|z|<r_{m}^{\prime \prime} / 4 \equiv R_{m}$ and $W_{m}(0)=0$, it is the real part of a regular function $f_{m}(z)$ which may be taken to satisfy $f_{m}(0)=0$. Let

$$
f_{m}(z)=\sum_{n=1}^{\infty} C_{n}\left(R_{m}\right) z^{n} \quad\left(|z|<R_{m}\right)
$$

Then

$$
\begin{aligned}
C_{n}\left(R_{m}\right) r^{n}= & \frac{1}{\pi} \int_{-\pi}^{+\pi} W_{m}\left(r e^{i \theta}\right) e^{-\imath n \theta} d \theta\left(r<R_{m}, n \geqq 1\right) . \\
= & \frac{1}{\pi} \int_{-\pi}^{+\pi} v\left(r e^{i \theta}\right) e^{-\imath n \theta} d \theta-\frac{1}{\pi} \int_{-\pi}^{+\pi} u_{1, m}^{(1)}\left(r e^{i \theta}\right) e^{-\imath n \theta} d \theta+\frac{1}{\pi} \int_{-\pi}^{+\pi} u_{1, m}^{(2)}\left(r e^{\imath \theta}\right) e^{-\imath n \theta} d \theta \\
= & \frac{1}{\pi} \int_{-\pi}^{+\pi} v\left(r e^{i \theta}\right) e^{-\imath n \theta} d \theta+\frac{1}{n} \int_{|\zeta| \leqq r}\left(\frac{\bar{\zeta}}{r}\right)^{n} d \nu^{(1)}(\zeta)+\frac{1}{n} \int_{r<|\zeta| \leqq R_{m}}\left(\frac{r}{\zeta}\right)^{n} d \nu^{(1)}(\zeta) \\
& -\frac{1}{n} \int_{|\zeta| \leqq r}\left(\frac{\bar{\zeta}}{r}\right)^{n} d \nu^{(2)}(\zeta)-\frac{1}{n} \int_{r<|\zeta| \leqq R_{m}}\left(\frac{r}{\zeta}\right)^{n} d \nu^{(2)}(\zeta) . \quad \text { (cf. [8]) }
\end{aligned}
$$

Evidently

$$
\left|\frac{1}{\pi} \int_{-\pi}^{+\pi} v\left(r e^{i \theta}\right) e^{-\imath n \theta} d \theta\right| \leqq 2 N(r,|v|) \leqq 4 T(r, v)-2 N\left(r, u^{(1)}\right)-2 N\left(r, u^{(2)}\right),
$$




$$
\left|\int_{|\zeta| \leqq r}\left(\frac{\bar{\zeta}}{r}\right)^{n} d \nu^{(1)}(\zeta)\right| \leqq \int_{0}^{r} d n^{(1)}(t)=n^{(1)}(r), \quad \text { etc. }
$$

Hence by (45) we have

$$
\left|C_{n}\left(R_{m}\right)\right| \leqq \frac{4 T\left(R_{m}, v\right)}{R_{m}^{n}}+\frac{n^{(1)}\left(R_{m}\right)+n^{(2)}\left(R_{m}\right)}{n \cdot R_{m}^{n}}
$$

$$
\leqq \frac{4 T\left(2 R_{m}, v\right)}{R_{m}^{n}}+\frac{2 T\left(2 R_{m}, v\right)}{n \cdot R_{m}^{n} \cdot \log 2}
$$

Substituting (46) into (44), we obtain for $r=|z|<R_{m} / 2$

$$
\begin{aligned}
\left|W_{m}(z)\right| & \leqq\left|f_{m}(z)\right| \leqq \sum_{n=1}^{\infty}\left|C_{n}\left(R_{m}\right)\right| r^{n} \\
& <4 T\left(2 R_{m}, v\right) \sum_{n=1}^{\infty}\left(\frac{r}{R_{m}}\right)^{n}+2 T\left(2 R_{m}, v\right) \frac{1}{\log 2} \sum_{n=1}^{\infty}\left(\frac{r}{R_{m}}\right)^{n} \\
& =4 T\left(2 R_{m}, v\right) \frac{r / R_{m}}{1-r / R_{m}}+2 T\left(2 R_{m}, v\right) \frac{1}{\log 2} \frac{r / R_{m}}{1-r / R_{m}} \\
& <\frac{T\left(2 R_{m}, v\right)}{R_{m}} r\left(8+\frac{4}{\log 2}\right) .
\end{aligned}
$$

From (43), (16), (47) and (42), we deduce that for any $\eta>0$ and any integer $s>0$, there exists a $m_{0}=m_{0}(\eta, s)>0$ such that $r \in I_{m}(s), m \geqq m_{0}$ imply

$$
m^{*}(r, v) \geqq u_{2, m}^{(1)}(-r)-u_{2, m}^{(2)}(r)-\eta V(r) .
$$

Now, for given $\eta>0$, choose an integer $s>0$ such that $h_{s}(\pi)>h(\pi)-\eta$. By the definition of $h_{s}(\pi)$ there exists a sequence $\left\{\chi_{n}\right\}_{1}^{\infty} \subset \Lambda(s)$ tending to $\infty$ such that

$$
B\left(-\chi_{n}\right)>\left(h_{s}(\pi)-\eta\right) V\left(\chi_{n}\right)>(h(\pi)-2 \eta) V\left(\chi_{n}\right)>(h(0) \cos \pi \rho-2 \eta) V\left(\chi_{n}\right) .
$$

We may suppose that $\cos \pi \rho-2 \eta>0$. By (48) and (49) we have

$$
m^{*}\left(\chi_{n}, v\right)>(h(\pi)-3 \eta) V\left(\chi_{n}\right)-\left\{u_{2, m}^{(2)}\left(-\chi_{n}\right)+u_{2, m}^{(2)}\left(\chi_{n}\right)\right\} .
$$

Since $N\left(r, u^{(1)}\right) \sim T(r, v)(r \rightarrow \infty)$, we obtain for any $\varepsilon>0$,

$$
N\left(r, u_{2, m}^{(2)}\right) \sim N\left(r, u^{(2)}\right)<(1-\delta(\infty, v)+\varepsilon) N\left(r, u^{(1)}\right) \quad\left(r \in I_{m}(s), r \geqq r_{0}(\varepsilon)\right) .
$$

As we have shown in the proof of Theorem 1,

$$
u_{2, m}^{(2)}(r)=r \int_{0}^{\infty} \frac{N\left(t, u_{2, m}^{(2)}\right)}{(t+r)^{2}} d t
$$

Using this and (51), we easily have for $r \in I_{m}(s), m>m_{0}(\eta, s)$ 


$$
u_{2, m}^{(2)}(r)<(1-\delta(\infty, v)) u_{1, m}^{(2)}(r)+\eta V(r) .
$$

Thus for $r \in I_{m}(s), m>m_{0}(\eta, s)$

$$
\begin{aligned}
u_{2, m}^{(2)}(-r)+u_{2, m}^{(2)}(r) \leqq 2 u_{2, m}^{(2)}(r) \leqq & \left(\frac{2-2 \delta(\infty, v)}{2-\delta(\infty, v)}+\eta^{\prime}\right)\left(u_{2, m}^{(1)}(r)+u_{2, m}^{(2)}(r)\right) \\
& <\left(\frac{2-2 \delta(\infty, v)}{2-\delta(\infty, v)}+\eta^{\prime \prime}\right) h(0) V(r),
\end{aligned}
$$

where $\eta^{\prime}, \eta^{\prime \prime}(>0)$ satisfy $\eta^{\prime}, \eta^{\prime \prime} \rightarrow 0$ as $\eta \rightarrow 0$. Substituting (52) into (50),

$$
m^{*}\left(\chi_{n}, v\right)>\left\{h(\pi)-3 \eta h(0)-\frac{2-2 \delta(\infty, v)}{2-\delta(\infty, v)} h(0)-\eta^{\prime \prime} h(0)\right\} V\left(\chi_{n}\right)
$$

We may suppose that the right hand side of (53) is positive. On the other hand, by (43) and (47),

$$
\begin{aligned}
m_{2}^{2}\left(\chi_{n}, v\right)= & m_{2}^{2}\left(\chi_{n}, u_{1, m}^{(1)}-u_{1, m}^{(2)}\right)+\frac{1}{2 \pi} \int_{0}^{2 \pi}\left(W_{m}\left(\chi_{n} e^{\imath \theta}\right)\right)^{2} d \theta \\
& +2 \frac{1}{2 \pi} \int_{0}^{2 \pi} W_{m}\left(\chi_{n} e^{\imath \theta}\right)\left(u_{1, m}^{(1)}\left(\chi_{n} e^{\imath \theta}\right)-u_{1, m}^{(2)}\left(\chi_{n} e^{\imath \theta}\right)\right) d \theta \\
\leqq & m_{2}^{2}\left(\chi_{n}, u_{1, m}^{(1)}-u_{1, m}^{(2)}\right)+\eta^{2}\left(V\left(\chi_{n}\right)\right)^{2}+2 m_{2}\left(\chi_{n}, u_{1, m}^{(1)}-u_{1, m}^{(2)}\right) \eta V\left(\chi_{n}\right) .
\end{aligned}
$$

As Miles and Shea [11] proved,

$$
m_{2}\left(\chi_{n}, u_{1, m}^{(1)}-u_{1, m}^{(2)}\right) \leqq m_{2}\left(\chi_{n}, u_{2, m}^{(1)}+u_{2, m}^{(2)}\right)
$$

Here we note that for $|z|=\chi_{n}$

$$
0<u_{2, m}^{(1)}(z)+u_{2, m}^{(2)}(z)<(H(\theta)+\varepsilon) V\left(\chi_{n}\right),
$$

where

$$
H(\theta)=\frac{h(0) \sin (\pi-\theta) \rho+h(\pi) \sin \theta \rho}{\sin \pi \rho} \quad(0 \leqq \theta \leqq \pi) .
$$

Hence

$$
\begin{aligned}
& m_{2}\left(\chi_{n}, u_{2, m}^{(1)}+u_{2, m}^{(2)}\right)<V\left(\chi_{n}\right)\left\{\frac{1}{\pi} \int_{0}^{\pi}(H(\theta)+\varepsilon)^{2} d \theta\right\}^{1 / 2} \\
& <V\left(\chi_{n}\right)\left\{\frac{\left[\left\{(h(0))^{2}+(h(\pi))^{2}\right\}\left(\frac{1}{2}-\frac{\sin 2 \pi \rho}{4 \pi \rho}\right)+h(0) h(\pi)\left(\frac{\sin \pi \rho}{\pi \rho}-\cos \pi \rho\right)\right]^{1 / 2}}{\sin \pi \rho}+\eta^{\prime \prime \prime}\right\},
\end{aligned}
$$

where $\eta^{\prime \prime \prime} \rightarrow 0$ as $\varepsilon \rightarrow 0$. Combining (53)-(56), we obtain 


$$
\begin{aligned}
& \frac{m^{*}\left(\chi_{n}, v\right)}{m_{2}\left(\chi_{n}, v\right)} \\
> & \frac{\left(h(\pi)-\frac{2-2 \delta(\infty, v)}{2-\delta(\infty, v)} h(0)-3 \eta h(0)-\eta^{\prime \prime} h(0)\right) \sin \pi \rho}{\left[\left\{(h(0))^{2}+(h(\pi))^{2}\right\}\left(\frac{1}{2}-\frac{\sin 2 \pi \rho}{4 \pi \rho}\right)+h(0) h(\pi)\left(\frac{\sin \pi \rho}{\pi \rho}-\cos \pi \rho\right)\right]^{1 / 2}+\eta^{\prime \prime \prime}} .
\end{aligned}
$$

The function

$$
\frac{t-\frac{2-2 \delta(\infty, v)}{2-\delta(\infty, v)}}{\left\{\left(1+t^{2}\right)\left(\frac{1}{2}-\frac{\sin 2 \pi \rho}{4 \pi \rho}\right)+t\left(\frac{\sin \pi \rho}{\pi \rho}-\cos \pi \rho\right)\right\}^{1 / 2}}
$$

increases as $t$ increases, and therefore, in view of $h(\pi)>h(0) \cos \pi \rho$, the right hand side of (57) is not smaller than

$$
\frac{\cos \pi \rho-\frac{2-2 \delta(\infty, v)}{2-\delta(\infty, v)}}{\sqrt{ } 1 / 2+\sin 2 \pi \rho / 4 \pi \rho}-\varepsilon .
$$

Proof of Corollary 3. We make use of some estimates stated in $\S 4$. As Valiron [13] showed

$$
\sum_{j=1}^{N} \log ^{+}\left|y_{j}\right| \leqq \max _{0 \leqq j \leqq N} \log \left|A_{j} / A_{0}\right|+O(1) .
$$

Hence

$$
C^{2}(r, y) \leqq\left[\frac{1}{2 \pi} \int_{-\pi}^{+\pi}\left\{\max _{0 \leqq J \leqq N} \log \left|\frac{A_{\jmath}}{A_{0}}\left(r e^{\imath \theta}\right)\right|\right\}^{2} d \theta\right](1+o(1))
$$

We apply Theorem 3 to $u^{(1)}=\max _{0 \leq j \leq N} \log \left|A_{\jmath}\right|, u^{(2)}=\log \left|A_{0}\right|$. Then there exist an integer $s=s(\varepsilon)>0$ and a sequence $\left\{\chi_{n}\right\}_{1}^{\infty} \subset \Lambda(s)$ tending to $\infty$ such that

$$
\begin{aligned}
& \left\{\min _{|z|=\chi_{n}} \max _{1 \leq j \leq N} \log \left|A_{j} / A_{0}\right|\right\}^{2} \\
> & \left\{\frac{k_{2}}{\sqrt{1 / 2+\sin 2 \pi \rho / 4 \pi \rho}}-\varepsilon / 2\right\}^{2}\left[\frac{1}{2 \pi} \int_{-\pi}^{+\pi}\left\{\max _{0 \leqq j \leqq N} \log \left|\frac{A_{\jmath}}{A_{0}}\left(\chi_{n} e^{i \theta}\right)\right|\right\}^{2} d \theta\right] .
\end{aligned}
$$

Combining (58), (59) and an estimate stated in $\S 4$, we have

$$
N \log m^{*}\left(\chi_{n}, y\right)>\left(\frac{k_{2}}{\sqrt{1 / 2+\sin 2 \pi \rho / 4 \pi \rho}}-\varepsilon\right) C\left(\chi_{n}, y\right) .
$$




\section{REFERENCES}

[1] Anderson, J. M. And Baernstein II, A., The size of the set on which a meromorphic function is large, Proc. London Math. Soc. (3) 36 (1978), 518-539.

[2] Baernstein II, A., Proof of Edrei's spread conjecture, ibid, (3) 26 (1973), 418434.

[3] Baernstein II, A., A generalization of the $\cos \pi \rho$ theorem, Trans. Amer. Math. Soc. 193 (1974), 181-197.

[4] BARRY, P. D., On a theorem of Kjellberg, Quart. J. Math. Oxford (2) 15 (1964), 179-191.

[5] Cartwright, M. L., Integral functions (Cambridge, 1956).

[6] Drasin, D. And Shea, D.F., Pólya peaks and the oscillation of positive functions Proc. Amer. Math. Soc. 34 no. 2 (1972), 403-411.

[7] EDREI, A., A local form of the Phragmén-Lindelöf indicator; Mathematika 17 (1970), 149-172.

[8] EDREI, A. AND Fuchs, W. H.J., On the growth of meromorphic functions with several deficient values, Trans. Amer. Math Soc. 93 (1959), 292-328.

[9] Hayman, W.K. And Kennedy, P.B., Subharmonic functions, Academic Press (1976).

[10] KJellberg, B., On the minimum modulus of functions of lower order less than one, Math. Scand. 8 (1960), 189-197.

[11] Miles, J. And Shea, D.F., An extremal problem in value distribution theory, Quart. J. Math. Oxford (2) 24 (1973), 377-383.

[12] Ozawa, M., On the minimum modulus of an entire algebroid function of lower order less than one; Kōdai Math. Sem. Rep. 22 (1970), 166-171.

[13] Valiron, G., Sur la dérivée des functions algébroides, Bull. Soc. Math. 59 (1931), 17-39.

Department of Mathematics,

Tokyo Institute of Technology

Current Address

Department of Mathemattcs,

Daido Institute of Technology,

Daido-cho, Minami-ku, Nagoya, Japan 